\title{
BIFURCATION ANALYSIS OF THE DYNAMICAL SYSTEM FOR A THREE- LAYERED VALVE WITH PERPENDICULAR ANISOTROPY
}

\author{
Natalia Ostrovskaya ${ }^{1, *}$, Vladimir Skidanov ${ }^{1}$, Iulia Iusipova $^{1,2}$, and Maxims Skvortsov ${ }^{1,2}$ \\ ${ }^{1}$ Institute for Design Problems in Microelectronics, Department of Microelectronic Components, Soviet Str. 3, Zelenograd, 124365 \\ Moscow, Russia \\ ${ }^{2}$ National Research University of Electronic Technology, Department of Applied Mathematics, Zelenograd, Bld.1, Shokin Square, \\ 124498, Moscow, Russia
}

\begin{abstract}
The features of switching dynamics in a model of a three-layered valve have been investigated theoretically and numerically. For this purpose, the system of ordinary differential equations in the approximation of the uniform magnetization distribution for the magnetization dynamics in the valve with perpendicular anisotropy was derived. It was shown that in such a system, in contrast with the system for the in-plane anisotropy, there are only two equilibrium positions of the magnetization vector. The stability analysis of the stationary points of the system has been carried out. With its help, the classification of types of dynamics versus field and current values was performed. The regions of limit cycles existence and the regions of optimal magnetization switching were revealed.
\end{abstract}

The magnetic random access memory (MRAM) attracts the attention of electronics engineers due to its high speed of magnetic switching, low energy consumption, high data storage density, and reliability. In the basic publication by J. Slonczewski (1996) [1], the first suggested model is composed of two ferromagnetic layers with in-plane anisotropy separated by a nonmagnetic interlayer. However, both calculations and experiments showed that switching currents in the classical ferromagnetics, such as cobalt and iron, are too high. It was found experimentally that the most optimal materials for the ferromagnetic layers are the ferromagnetic alloys $\mathrm{FeCoB}$, and the most promising design solution is a memory cell with perpendicular magnetic anisotropy of the ferromagnetic layers [3-7].

\section{Model}

The object under study is a three-layered valve structure consisting of two ferromagnetic layers separated by nonmagnetic one. The cross-section of the structure is the $d_{s} \times d_{s}$ square; the thickness of the thin layer is $d_{1}$. These dimensions assumed to be small enough to use the uniform distribution of the magnetization in the layer. The magnetization of the reference (thick) layer is fixed and directed from the thick to the thin layer. This direction is taken as positive for the OZ-axis, which is perpendicular to the layers. The current passes in parallel to OZ-axis. Its density $J$ lies in the interval from 0 to $10^{13} \mathrm{~A} / \mathrm{m}^{2}$. The structure is placed into the external magnetic field parallel to $\mathrm{OZ}$. This field can be either positive or negative (Fig. 1).

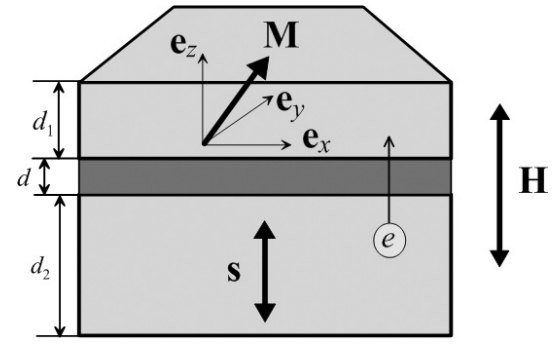

Fig. 1. Geometry of the model.

\subsection{Basic Equations}

The theory of the phenomenon is proposed by J. Slonczewski [1]. The model is based on the fundamental Landau-Lifshits-Gilbert equation that describes the dynamics of the magnetization vector $\mathbf{M}$ in the free ferromagnetic layer:

$$
\frac{\partial \mathbf{M}}{\partial t}=-|\gamma| \mu_{0}\left[\mathbf{M} \times \mathbf{H}^{\mathrm{eff}}\right]+\frac{\alpha}{M_{\mathrm{s}}}\left[\mathbf{M} \times \frac{\partial \mathbf{M}}{\partial t}\right] .
$$

Here $\mu_{0}=4 \pi \cdot 10^{-7} \mathrm{~N} / \mathrm{A}^{2}$ is the magnetic constant, $\gamma=1.76 \cdot 10^{11} \mathrm{rad} \cdot \mathrm{T}^{-1} \cdot \mathrm{s}^{-1}$ is the gyromagnetic ratio, $\alpha$ is the dimensionless damping parameter, $M_{s}$ is the saturation magnetization, $\mathbf{H}^{\text {eff }}$ is the effective magnetic field, which is the equivalent of those physical interactions that are taken into account in the model. In the case under consideration, it is

$$
\mathbf{H}^{\text {eff }}=\mathbf{H}+\mathbf{H}_{\mathrm{a}}+\mathbf{H}_{\mathrm{f}}+\mathbf{H}_{\mathrm{c}},
$$

\footnotetext{
Corresponding author: n.ost@ippm.ru
} 
where $\mathbf{H}$ is the external magnetic field, $\mathbf{H}_{\mathbf{a}}$ is the effective field of the magnetic anisotropy, $\mathbf{H}_{\mathrm{f}}$ is the effective demagnetizing field that appears because of the finite sizes of the valve, $\mathbf{H}_{c}$ is the effective field originated due to the spin-polarized injection current. The exchange interaction is ignored due to the small sizes of the structure (the approximation of uniform magnetization). We supposed the anisotropy effective field and the magnetization of the fixed layer to be perpendicular to the valve layers. At that, the magnetization of the fixed layer is supposed to be directed to the free layer of the valve (the vector $\mathbf{S}$ in Fig. 1, $|\mathbf{s}|=1$ ). The magnetization of the free layer at the first moment is taken to be co-directed with the magnetization of the fixed layer (parallel orientation). After the simultaneous field and current engaging, it can pass to another stationary position. It is natural to name the parallel orientation of the magnetization vectors as "zero", and the anti-parallel orientation as "one" (or vice versa).

form

Equation (1) can be written in the dimensionless

$$
\frac{\partial \mathbf{m}}{\partial \tilde{\tau}}=-\left[\mathbf{m} \times \mathbf{h}^{\mathrm{eff}}\right]+\alpha\left[\mathbf{m} \times \frac{\partial \mathbf{m}}{\partial \tilde{\tau}}\right],
$$

where $\mathbf{m}=\frac{\mathbf{M}}{M_{s}}, \mathbf{h}^{\text {eff }}=\frac{\mathbf{H}^{\text {eff }}}{M_{\mathrm{s}}},|\mathbf{m}|=1$, time $\tilde{\tau}$ is measured in the units $\left(\gamma \mu_{0} M_{s}\right)^{-1}$, and $\mathbf{h}^{\text {eff }}=\mathbf{h}+\mathbf{h}_{\mathrm{a}}+\mathbf{h}_{\mathrm{f}}+\mathbf{h}_{\mathrm{c}}$.

1) In the general case, the external field $\mathbf{h}$ can be presented as

$$
\mathbf{h}=h_{x} \mathbf{e}_{x}+h_{y} \mathbf{e}_{y}+h_{z} \mathbf{e}_{z} .
$$

In the case under consideration, the external field is parallel to OZ-axis, i.e.

$$
\mathbf{h}=h \mathbf{e}_{z} .
$$

2) The anisotropy field is also oriented along OZ-axis

$$
\mathbf{h}_{\mathrm{a}}=k\left(\mathbf{m}, \mathbf{e}_{z}\right) \mathbf{e}_{z}=k m_{z} \mathbf{e}_{z},
$$

where $k=2 K_{a} \mu_{0}^{-1} M_{\mathrm{s}}^{-2}$, and $K_{a}$ is the anisotropy constant.

3) The demagnetizing field $\mathbf{h}_{\mathrm{f}}$ is defined as $\mathbf{h}_{\mathrm{f}}=-\hat{\mathbf{q}} \mathbf{m}$, where $\hat{\mathbf{q}}$ is a tensor (form-factor). In the geometry of this model we can put (see [2], for example)

$$
\mathbf{h}_{\mathrm{f}}=-m_{z} \mathbf{e}_{\mathbf{z}} \text {. }
$$

4) According to the Slonczewski-Berger theory, the contribution into effective field caused by the spinpolarized current is

$$
\mathbf{h}_{\mathrm{c}}=G \frac{J}{J_{n}}[\mathbf{s} \times \mathbf{m}],
$$

where $\mathbf{s}$ is the unit vector of the spin polarization, the direction of which is coincident with the direction of the magnetization in the thick layer (in this geometry $\left.\mathbf{s} \equiv \mathbf{e}_{z}\right), \quad J$ is the dimensional density of the spinpolarized current, $J_{n}$ is the normalization coefficient, which is equal to

$$
J_{n}=\frac{d_{1} e \mu_{0} M_{s}^{2}}{\hbar},
$$

( $\hbar$ is the Plank constant, $\mu_{0}$ is the magnetic constant, $e$ is the charge of the electron, $d_{1}$ is the thickness of the free ferromagnetic layer). Hence, the dimensionless current density is $j=J / J_{n}$. According to [1], the scalar dimensionless function $G(\mathbf{m})$ is as follows:

$$
G=\frac{4 P^{3 / 2}}{(1+P)^{3}(3+(\mathbf{s}, \mathbf{m}))-16 P^{3 / 2}},
$$

where $P$ is the parameter of the spin polarization in the interface of the valve.

Taking into account that, in this case, $\mathbf{s} \equiv \mathbf{e}_{\mathrm{z}}$, we obtain $(\mathbf{s}, \mathbf{m})=m_{z}$. Hence,

$$
G=\frac{4 P^{3 / 2}}{(1+P)^{3}\left(3+m_{z}\right)-16 P^{3 / 2}}=\frac{c}{b+m_{z}},
$$

$\left(c=4 P^{3 / 2} /(1+P)^{3}, b=3-4 c\right)$. Note that, in our geometry, $[\mathbf{s} \times \mathbf{m}]=-m_{y} \cdot \mathbf{e}_{\mathrm{x}}+m_{x} \mathbf{e}_{\mathrm{y}}$. Therefore, for the valve with the perpendicular anisotropy, the effective magnetic field takes the following form:

$$
\mathbf{h}^{\text {eff }}=\left(\begin{array}{l}
h_{x}^{\text {eff }} \\
h_{y}^{\text {eff }} \\
h_{z}^{\text {eff }}
\end{array}\right)=\left(\begin{array}{l}
-g m_{y} \\
g m_{x} \\
k m_{z}-m_{z}+h
\end{array}\right),
$$

where $g=G j$.

After certain algebraic transformations of (3), we obtain

$$
\frac{\partial \mathbf{m}}{\partial \tau}=-\left[\mathbf{m} \times \mathbf{h}^{\text {eff }}\right]+\alpha \mathbf{h}^{\text {eff }}-\alpha \mathbf{m}\left(\mathbf{m}, \mathbf{h}^{\text {eff }}\right),
$$

where $\tau=\frac{\tilde{\tau}}{1+\alpha^{2}}=|\gamma| \frac{\mu_{0} M_{s}}{1+\alpha^{2}} t$. In the coordinate form, the vector equation (6) is equivalent to the system

$$
\begin{array}{r}
\frac{d m_{x}}{d t}=j \frac{c}{b+m_{z}} m_{x} m_{z}-m_{y}\left(k m_{z}-m_{z}+h\right)-\alpha j \frac{c}{b+m_{z}} m_{y}- \\
-\alpha m_{x} m_{z}\left(k m_{z}-m_{z}+h\right), \\
\begin{array}{r}
\frac{d m_{y}}{d t}=m_{x}\left(k m_{z}-m_{z}+h\right)+j \frac{c}{b+m_{z}} m_{y} m_{z}+\alpha j \frac{c}{b+m_{z}} m_{x}- \\
-\alpha m_{y} m_{z}\left(k m_{z}-m_{z}+h\right), \\
\frac{d m_{z}}{d t}=-j \frac{c}{b+m_{z}} m_{y}^{2}-j \frac{c}{b+m_{z}} m_{x}^{2}+\alpha\left(k m_{z}-m_{z}+h\right)- \\
-\alpha m_{z}^{2}\left(k m_{z}-m_{z}+h\right) .
\end{array}
\end{array}
$$

The parameters of the $\mathrm{Co} / \mathrm{Cu} / \mathrm{Co}$ structure, which were used in the numerical calculations, were $\alpha=0.02$, $P=0.35, \quad \mu_{0} M_{s}=1.76 \mathrm{~T}, \quad K_{a}=0.53 \cdot 10^{6} \mathrm{~J} / \mathrm{m}^{3}$, the thickness of the free layer was $d_{1}=4 \mathrm{~nm}$. The normalization coefficients were as follows:

$$
\begin{aligned}
& t=\frac{1+\alpha^{2}}{\gamma \mu_{0} M_{s}} \tilde{\tau} \cong 2 \cdot 10^{-10} \tau(\mathrm{s}), \\
& K_{a}=\frac{\mu_{0} M_{s}^{2}}{2} k \cong 1.2 \cdot 10^{6} k\left(\mathrm{~J} / \mathrm{m}^{3}\right), \\
& H=M_{s} h \cong 1.4 \cdot 10^{6} h(\mathrm{~A} / \mathrm{m}), \\
& J=\frac{d_{1} e \mu_{0} M_{s}^{2}}{\hbar} j \cong 1.9 \cdot 10^{13} j\left(\mathrm{~A} / \mathrm{m}^{2}\right) .
\end{aligned}
$$




\section{Stationary points}

The first step to the qualitative classification of the dynamical regimes in the system (7) is to find the equilibrium states of the magnetization in the free layer of the valve. With this purpose, the right-hand parts of (7) must be equated to zero:

$$
\left\{\begin{array}{l}
\left(m_{z} h_{y}-m_{y} h_{z}\right)+\alpha h_{x}-\alpha m_{x} L=0 \\
\left(m_{x} h_{z}-m_{z} h_{x}\right)+\alpha h_{y}-\alpha m_{y} L=0 \\
\left(m_{y} h_{x}-m_{x} h_{y}\right)+\alpha h_{z}-\alpha m_{z} L=0
\end{array}\right.
$$

where $L=(\mathbf{m}, \mathbf{h})$. As a result, we obtain the algebraic fractionally-rational set of three equations for three variables $m_{x}, m_{y}, m_{z}$. Parameters $\alpha, P, k$ are thought to be fixed (internal), whereas the field $h$ and the current density $j$ are scalable (in other words, external or control ones). Solutions $m_{x}, m_{y}, m_{z}$ of (8) at the current values $h, j$ are nothing else but the stationary points of (7). At $h=0, j=0$, the system (8) degenerates to the form

$$
\left\{\begin{array}{l}
m_{y} m_{z}(1-k)-\alpha m_{x} L=0 \\
(k-1) m_{x} m_{z}-\alpha m_{y} L=0 \\
\alpha m_{z}(k-1)-\alpha m_{z} L=0 .
\end{array}\right.
$$

Here, $L=(k-1) m_{z}^{2}$, and the system (7) has two stationary points on the surface of the unit sphere with coordinates $T_{1,2}(0,0, \pm 1)$, and the singular line, which coincides with the equator of the sphere.

To find the singular points at the nonzero external parameters, it is necessary to solve (8) in the general form. It is not difficult to show that it has no other roots, except $T_{1,2}(0,0, \pm 1)$.

\subsubsection{Type and stability of singular points}

1) Point $T_{1}(0,0,+1)$.

The matrix of the system (7) linearized in the vicinity of the singular point $T_{1}(0,0,+1)$ has the form

$$
\begin{aligned}
& \left.\mathbf{A}\right|_{T_{1}}= \\
& \left(\begin{array}{ccc}
\frac{j c-\alpha(b+1)(h-1+k)}{b+1} & -\frac{\alpha j c+(b+1)(h-1+k)}{b+1} & 0 \\
\frac{\alpha j c+(b+1)(h-1+k)}{b+1} & \frac{j c-\alpha(b+1)(h-1+k)}{b+1} & 0 \\
0 & 0 & -2 \alpha(h-1+k)
\end{array}\right) .
\end{aligned}
$$

Its eigenvalues are

$$
\begin{aligned}
& \lambda_{1,2}^{+}=\frac{j c-\alpha(b+1)(h-1+k)}{b+1} \pm i \frac{\alpha j c+(b+1)(h-1+k)}{b+1}, \\
& \lambda_{3}^{+}=-2 \alpha(h-1+k) .
\end{aligned}
$$

The product of the eigenvalues $\lambda_{1}^{+}$and $\lambda_{2}^{+}$is

$$
\lambda_{1}^{+} \lambda_{2}^{+}=\frac{\left(1+\alpha^{2}\right)}{(b+1)^{2}}\left[(b+1)^{2}(h-1+k)^{2}+j^{2} c^{2}\right] \geq 0 \text {, }
$$

therefore, this singular point cannot be of a saddle-type, but is only a focus or a node.

Analyzing the eigenvalues, we see two threshold relations for the point $T_{1}(0,0,+1)$

$$
\begin{aligned}
& L_{1}: \quad \alpha c j+(b+1) h-(b+1)(1-k)=0, \\
& L_{2}: \quad c j-\alpha(b+1) h+\alpha(b+1)(1-k)=0 .
\end{aligned}
$$

In the plane of the control parameters $(h, j)$, line $L_{1}$ separates the regions II and III, where the directions of the trajectories rotation in the vicinity of the singular point are opposite. Exactly in the line $L_{1}$, the singular point degenerates into a node.

Line $L_{2}$ separates the regions of the focus stability (I) and instability (II), therefore, according to the Andronov-Hopf theorem, in the vicinity of this line, a limit cycle must exist. It really does exist under the line $L_{2}$ and is unstable. The bifurcation diagram for the singular point $T_{1}(0,0,+1)$ is shown in Fig. 2 a.

2) Point $T_{2}(0,0,-1)$

The matrix of the system linearized in the vicinity of this point is

$$
\begin{aligned}
& \left.\mathbf{A}\right|_{T_{2}}= \\
& \left(\begin{array}{ccc}
\frac{j c+\alpha(1-b)(h+1-k)}{1-b} & \frac{\alpha j c-(1-b)(h+1-k)}{1-b} & 0 \\
-\frac{\alpha j c-(1-b)(h+1-k)}{1-b} & \frac{j c+\alpha(1-b)(h+1-k)}{1-b} & 0 \\
0 & 0 & 2 \alpha(h+1-k)
\end{array}\right) .
\end{aligned}
$$

The eigenvalues are as follows:

$$
\begin{aligned}
& \lambda_{1,2}^{-}=\frac{-j c+\alpha(b-1)(h+1-k)}{b-1} \pm i \frac{\alpha j c+(b-1)(h+1-k)}{b-1}, \\
& \lambda_{3}^{-}=2 \alpha(h+1-k) .
\end{aligned}
$$

As in the previous case, the product of $\lambda_{1}^{-}$and $\lambda_{2}^{-}$is always positive, because

$$
\lambda_{1}^{-} \lambda_{2}^{-}=\frac{\left(1+\alpha^{2}\right)}{(b-1)^{2}}\left[(b-1)^{2}(h+1-k)^{2}+j^{2} c^{2}\right] \geq 0 .
$$

That is why, the same as the singular point $T_{1}(0,0,+1)$, the point $T_{2}(0,0,-1)$ cannot be of the saddle type. The equations of the critical lines, which separate the $(h, j)$ plane into the regions of the identical magnetization dynamics in the vicinity of $T_{2}(0,0,-1)$, are

$$
\begin{array}{ll}
L_{3}: & \alpha c j+(b-1) h+(b-1)(1-k)=0, \\
L_{4}: & c j-\alpha(b-1) h-\alpha(b-1)(1-k)=0 .
\end{array}
$$

Line $L_{3}$ separates the areas with opposite directions of the rotation of the trajectories around the point $T_{2}(0,0,-1)$ on the sphere. The points that belong to the line correspond to nodes. Line $L_{4}$ separates the regions of foci stability and instability. As above, this is the line of the appearance/disappearance of the limit cycle. The limit cycles exist below this line. The bifurcation diagram for the point $T_{2}(0,0,-1)$ is shown in Fig. $2 \mathrm{~b}$.

In the center of figure 3 , the superposition of the phase diagrams for the points $T_{1}$ and $T_{2}$ is displayed. 
Critical lines $L_{1}, L_{2}, L_{3}, L_{4}$ divide the $(h, j)$-plane into the regions of the total identical magnetization dynamics and the equivalent phase portraits. Note that, in the case of the opposite magnetization $\mathbf{s}$ of the reference layer, the common bifurcation diagram would be reflected from the vertical axis $j$.
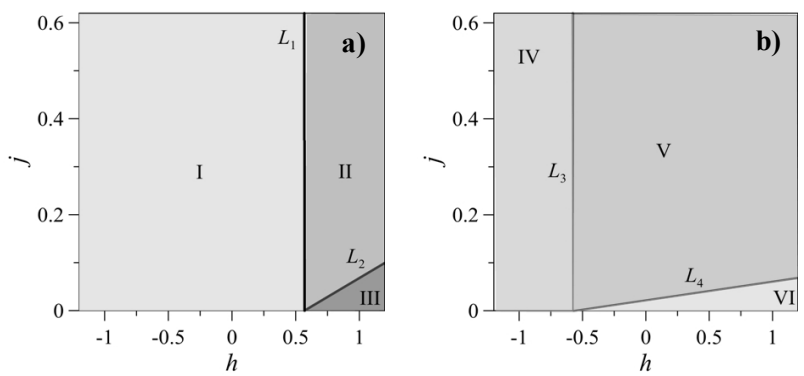

Fig. 2. Bifurcation diagrams: a) for the point $T_{1}(0,0,+1)$, b) for the point $T_{2}(0,0,-1)$.

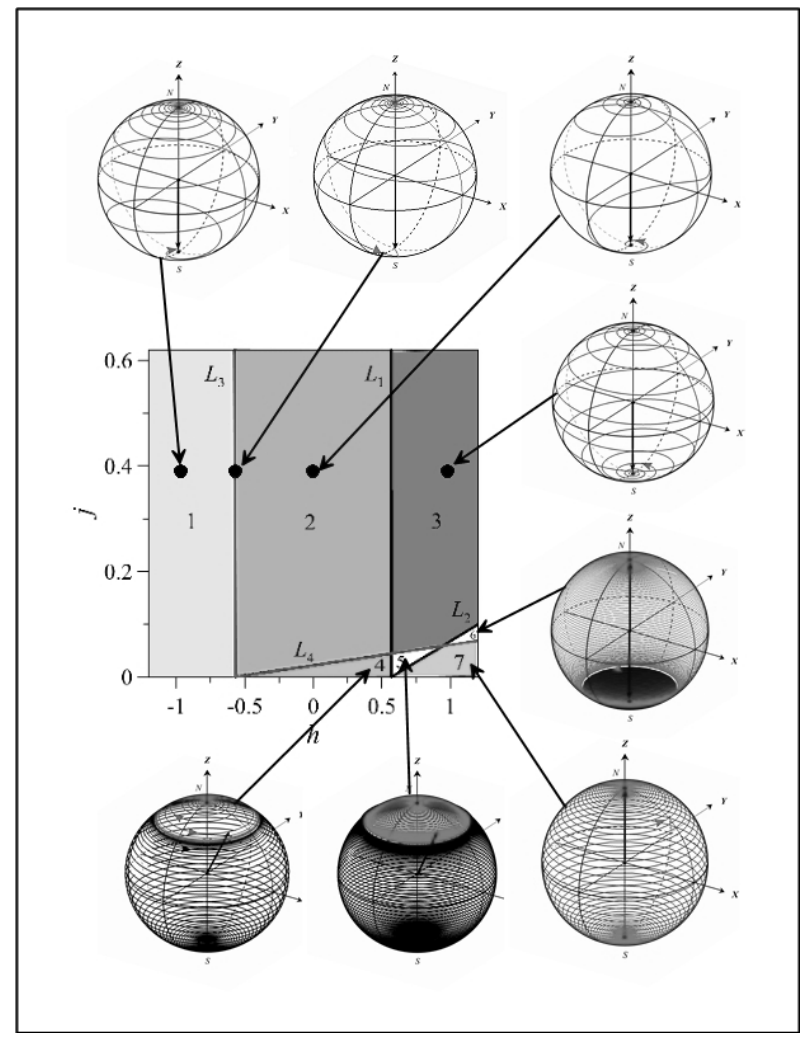

Fig. 3. Superposition of the bifurcation diagrams for the points $T_{1,2}(0,0, \pm 1)$ (in the center) and the examples of the magnetization hodographs for each region in the plane $(h, j)$.

\section{Results}

If the control parameters $h$ and $j$ belong to regions $1,2,3$, the singular point $T_{1}$ will be an unstable focus, although the direction of the vector rotation in these regions will be different. At the same time, the singular point $T_{2}$ will be a stable focus at these parameters. Therefore, at the parameters taken from these regions, the stable switching from parallel to antiparallel configuration takes place, which, for the parameters from region 2, happens with the change of the rotation direction (see the corresponding hodographs in Fig. 3). It should be noted that, at the same values of the current density, the speed of switching is higher in region 1 , than in 2 and 3.

For the parameters from region 4, the points $T_{1}$ and $T_{2}$ are the unstable foci with the opposite direction of the trajectory rotation. These points on the surface of the unit sphere are separated by the stable limit cycle. The trajectories started from $T_{1}$ and $T_{2}$ are attracted by this cycle. Let us note that the trajectories started from the point $T_{1}$ have the turning point, where they change the direction of rotation. Therefore, at the parameters belonging to region 4 , switching is impossible. This is true also for region 5 - the difference with region 4 is only in the absence of the turning point and, therefore, the unidirectional rotation of the magnetization for all the trajectories (Fig. 3). For the values of the field and the current from region 6 , the singular points $T_{1}$ and $T_{2}$ are the stable foci with the same direction of the trajectory rotation. They are separated by an unstable limit cycle (Fig. 3). Hence, in this region of the parameters, switching is impossible.

At the control parameters that belong to region 7 , the point $T_{1}$ is a stable focus, whereas the point $T_{2}$ is an unstable one with the constant direction of the trajectory rotation (Fig. 3). Here the reverse switching of the magnetization is possible, i.e., from the anti-parallel to parallel configuration. This switching occurs mainly under the influence of the magnetic field.

\section{References}

1. J. Slonczewski, J.Magn.Magn.Matter, 159, L1-L7 (1996).

2. R. Skomski, Simple models of Magnetism (Oxford University Press, 2012).

3. Yiming Huai, US PATENT No US 6,967,863 B2 Nov. 22 (2005).

4. Desin Wang, Haiwen Xi, Yuankai Zheng, D. Dimitar,// US PATENT Application Publication, Pub. No. US 2010/0109110 AI, May 6 (2010).

5. S. N. Piramanayagam, Chong, C. Tow, Developments in Data Storage: Materials and Perspective (A. John Wiley \& Sons, Inc., 2011)

6. S. Mangin, D. Ravelosona, Y. Henry, J. A. Katine, E. E. Fullerton, AAPPS Bulletin, 18, No.6, 41 (2008).

7. P. Kh. Amiri, Z. M. Zeng, J. Langer, H. Zhao et al., Appl.Phys.Lett. 98, 112507 (2011). 